\title{
Оптимизация параметров источников питания, возбуждаемых $\beta$-излучением
}

\author{
(C) С.В. Булярский ${ }^{1}$, А.В. Лакалин ${ }^{1}$, И.Е. Абанин ${ }^{2}$, В.В. Амеличев ${ }^{2}$, В.В. Светухин ${ }^{3}$ \\ ${ }^{1}$ Институт нанотехнологий микроэлектроники Российской академии наук \\ 119991 Москва, Россия \\ ${ }^{2}$ Научно-производственный комплекс „Технологический центр“, \\ 124498 Москва, Россия \\ ${ }^{3}$ Ульяновский государственный университет, \\ 432000 Ульяновск, Россия \\ E-mail: bulyar2954@mail.ru
}

(Получена 29 февраля 2016 г. Принята к печати 25 мартя 2016 г.)

\begin{abstract}
Представлены результаты экспериментальных исследований и расчетов эффективности преобразования энергии источников $\beta$-излучения Ni-63 в электрическую с помощью кремниевых $p-i-n$-диодов. Все вычисления выполнены с учетом распределения $\beta$-электронов по энергиям. Получено выражение для напряжения холостого хода преобразователя с учетом распределения электронов высоких энергий в области пространственного заряда $p-i-n$-диода. Показаны пути оптимизации параметров преобразователя за счет совершенствования технологии изготовления диодов, оптимизации толщины активного слоя излучателя и $i$-области полупроводникового преобразователя. Вычислено распределение потерь на преобразование источника и приемника излучения, а также потерь при входе электронов высоких энергий в полупроводник. Экспериментальные значения эффективности преобразования $0.4-0.7 \%$ хорошо согласуются с расчетными параметрами.
\end{abstract}

DOI: 10.21883/FTP.2017.01.43998.8223

\section{1. Введение}

Источники $\beta$-излучения обладают высокой удельной плотностью энергии [1]. Среди изотопов, применяемых в источниках питания, возбуждаемых $\beta$-излучением ( $\beta$-батареи), по запасам энергии лидируют Ni-63, тритий и $\mathrm{Sr}-90-3 \cdot 10^{5}-2 \cdot 10^{7}$ Вт $\cdot$ ч/кг. В связи с этим их выгодно применять для изготовления батарей $\mathrm{c}$ длительным сроком использования. Так, например, на основе изотопа Ni-63 можно создать источники питания со сроком службы более 30 лет [2,3]. Более высоким коэффициентом полезного действия обладают батареи, основанные на принципе прямого преобразования энергии. В этом случае $\beta$-электроны генерируют в полупроводнике электронно-дырочные пары, которые разделяются электрическим полем $p$-n-перехода или контакта металл-полупроводник [4-6]. Первоначально $\beta$-батареи изготавливались с использованием Pm-147 и трития, наносимых на плоскую поверхность диодных структур из кремния [6]. Недостатком изотопа Pm-147 является малый период полураспада (2.62 года). Это ограничивает срок службы батареек на его основе. Их использование не выгодно с экономической точки зрения. Кроме того, прометий трудно очистить от сопутствующих радиоактивных изотопов, создающих опасный фон $\gamma$-излучения. Конструкции с газообразным тритием имеют низкую электрическую мощность, получаемую с единицы объема преобразователя $\beta$-излучения. В связи с этим в $\beta$-батареях применяют гидриды металлов, в частности титана [1]. Такие батареи более компактны, но $\beta$-источники на основе гидридов обладают высоким самопоглощением электронов, возникающих при $\beta$-распаде. Глубина активной области, из которой электроны выходят на поверхность, всего 0.3-0.4 мкм. Поэтому их поверхностная активность - поток электронов, излучаемый в единицу времени с единицы площади поверхности, - мала, соответственно не высоки и электрические параметры $\beta$-батарей на основе гидридов и трития.

Изотоп никеля Ni-63 имеет период полураспада 100 лет. Невысокая средняя энергия $\beta$-электронов (17.6 кэВ) не создает проблем с радиационной защитой и настолько меньше порога радиационных повреждений полупроводника, что полностью исключает деградацию $p-n$-перехода. Поэтому этот изотоп, несмотря на его высокую стоимость, достаточно широко используется для изготовления $\beta$-батарей $[2,3,7,8]$. Недостатком конструкций на основе этого изотопа является низкий коэффициент полезного действия (кпд) $\beta$-батареи, который не превышает $1 \%$.

В данной работе анализируются пути повышения кпд $\beta$-батареи на основе источника излучения $\mathrm{Ni}-63$ и полупроводникового преобразователя - кремниевого $p-i-n$-диода. Рассматриваются возможности оптимизации размеров активных слоев источника и полупроводникового преобразователя, а также совершенствования технологии $p-i-n$-диода.

\section{2. Экспериментальные результаты, полученные с использованием $\beta$-источника на основе $\mathrm{Ni}-63$ и кремниевых $p-i-n$-диодов}

\section{1. Образцы для исследования}

В качестве преобразователя использовались $p-i-n$ диоды, с рабочей площадью $1 \mathrm{~cm}^{2}$. Исходным матери- 
алом для создания кристаллов $p-i-n$-диодов служили подложки высококачественного монокристаллического кремния марки 100 КЭФ-4400 (111). Слитки кремния с удельным сопротивлением 1000 Ом · см были выращены методом бестигельной зонной переплавки слитка, полученного по методу Чохральского. Согласно техническим условиям на пластины данной маркировки, плотность дефектов упаковки составляет не более $5 \cdot 10^{2} \mathrm{~cm}^{-2}$, а плотность дислокаций менее $1 \cdot 10^{2} \mathrm{~cm}^{-2}$.

Известно, что коэффициент преобразования $\beta$-излучения в электрический ток увеличивается при снижении обратных темновых токов. В технологию $p-i-n$-диодов были введены две технологические операции, а именно внутреннего геттерирования и создание охранного кольца. Геттерирование было реализовано путем формирования на обратной стороне кремниевой пластины сильно легированной области электронной проводимости. Эта область закрывалась слоями поликремния и нитрида кремния. После этого осуществлялся геттерирующий отжиг при температуре от 700 до $1000^{\circ} \mathrm{C}$. Выбор высококачественного кремния как исходного материала и введение указанных операций позволили снизить обратные токи в темноте при $295 \mathrm{~K}$ и напряжении обратного смещения 40 В практически в 10 раз: до 0.1-0.2 нА.

Конструктивно $\beta$-источник представляет собой металлическую подложку в виде прямоугольной пластины (фольги) из металлического никеля, на которую с одной стороны нанесен методом электрохимического осаждения радионуклид $\mathrm{Ni}-63$. Доля изотопа $\mathrm{Ni}-63$ a активном слое составляла $18 \%$, остальное приходилось на не радиоактивные атомы никеля. Активная часть герметизировалась тонким защитным слоем никеля. Размеры источника соответствовали рабочей поверхности $p-i-n$-диода. При наложении источника на диод он примыкал к поверхности достаточно плотно, практически не пропуская рассеянный свет. Между поверхностью диода и источником существовал небольшой воздушный зазор, который не играл существенной роли. При измерениях был использован набор источников излучения различной активности.

\section{2. Экспериментальные результаты преобразования энергии излучения в электрическую энергию}

Для всех диодов были измерены вольт-амперные характеристики при прямом и обратном смещении по стандартной методике в темноте. Далее эти результаты использовались для определения времен жизни электронов и дырок. Затем на диод накладывались источники $\beta$-излучения различной активности и измерение вольтамперных характеристик повторялось. При этом были определены напряжение холостого хода $\left(U_{\text {oc }}\right)$ и ток короткого замыкания $\left(I_{\mathrm{sc}}\right)$. Напряжение холостого хода при максимальных активностях изотопов достигало $120 \mathrm{MB}$ (рис. 1), а ток короткого замыкания менялся от 69 до 76 нА. В работах [7-9] с источником Ni-63 и преобразователем на основе кремниевых диодов достигнуты

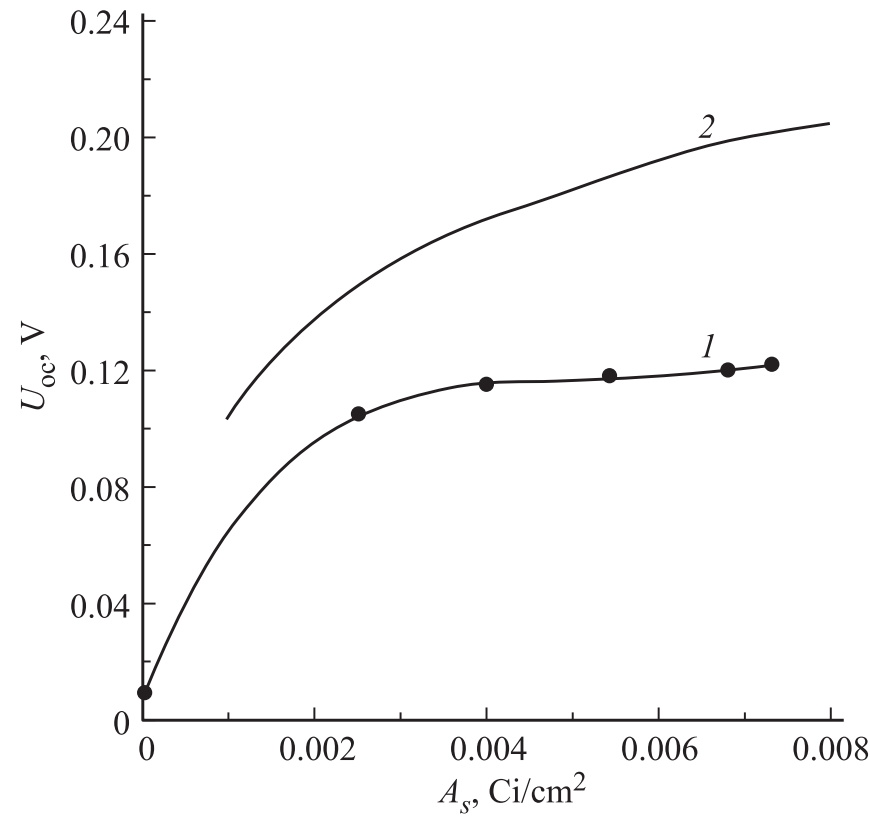

Рис. 1. Зависимость напряжения холостого хода от поверхностной активности источника излучения. 1 - эксперимент, 2 - расчет по формуле (19).

токи короткого замыкания от 2.8 до 50 нА при кпд менее $1 \%$. Это существенно ниже результатов данной работы. Рассчитаем кпд исследуемых диодов:

$$
\eta=P / E_{\mathrm{av}} A_{s} .
$$

Здесь $P$ - мощность, выделяемая при облучении диода на сопротивлении нагрузки; $E_{\mathrm{av}}-$ средняя энергия излучаемого $\beta$-электрона; $A_{s}$ - поверхностная активность источника излучения.

Расчеты дают максимальный кпд исследуемых диодов $0.8 \%$. Это значение превосходит полученные другими исследователями.

Далее проанализируем потери, которые снижают величину полезного тока $\beta$-батарейки.

\section{3. Оптимальная толщина $\beta$-источника}

Коэффициент полезного действия преобразователей определяется тремя основными процессами: самопоглощением, эффективностью сбора электронов и дырок, которые генерируются в области пространственного заряда полупроводниковой части преобразователя, и эффективностью полупроводниковой структуры $[1,10]$ :

$$
\eta=\eta_{\beta} \eta_{c} \eta_{s}
$$

Здесь $\eta_{\beta}$ - доля электронов, которые достигают поверхности полупроводникового преобразователя; $\eta_{c}$ доля электронов и дырок, которые попадают в область пространственного заряда и способны внести вклад в электрический ток; $\eta_{s}$ - интегральная эффективность диода, который обеспечивает конверсию. 
В данном пункте рассмотрим первый сомножитель. Электроны, выделяющиеся при $\beta$-распаде, имеют энергию десятки кэВ. Попадая в твердое тело, они теряют свою энергию на торможение. Существует несколько механизмов торможения, из которых нас интересует, в первую очередь, рассеяние с испусканием вторичных электронов, которые в дальнейшем вносят вклад в электрический ток источника питания. Для практических целей удобно воспользоваться формулой, которая аппроксимирует упомянутые расчеты, проведенные методом Монте-Карло [11]:

$$
h(\xi)=0.60+6.21 \xi-1204 \xi^{2}+5.23 \xi^{3}, \quad \xi=\frac{z}{R(E)},
$$

где $z$ - средняя глубина проникновения электрона в вещество; $R(E)$ - максимальная длина пробега по траектории электрона с энергией $E$, в материале с плотностью $\rho$. Для оценки этой величины используют формулу [12]:

$$
R(E)=\frac{0.0398}{\rho} E^{1.75} .
$$

Функция (3) имеет смысл вероятности обнаружения частицы с энергией $E$ на определенной глубине.

В изотопах достаточно сильно выражено явление самопоглощения, в результате которого на поверхность активного слоя попадают далеко не все электроны. Поэтому наращивание слоя изотопа не означает увеличение активности на его поверхности. Пусть активность $1 \mathrm{~cm}^{3}$ вещества равна $A_{0 V}$ (значение этой величины для Ni-63 равно $\left.3.1 \cdot 10^{13} \mathrm{c}^{-1} \cdot \mathrm{cm}^{-3}\right)$. Тогда активность слоя вещества толщиной $d z$, находящегося на глубине $z$ от поверхности, равна $A=A_{0 V} S d z$. Эта величина пропорциональна количеству электронов любой энергии, которые генерируются в этом слое.

Для расчета параметров активных слоев, как правило, используются средние значения энергии $\beta$-электронов. Такой подход недостаточно точен. В настоящей работе проводятся численные расчеты с использованием экспериментальных распределений, испускаемых при распаде электронов [13]. Энергия, которой обладает электрон при $\beta$-распаде, известна с некоторой вероятностью $W_{c}(E)$. Эту величину вычислим путем нормировки экспериментального распределения на единицу:

$$
A \int_{0}^{E_{\max }} f(E) d E=1 ; \quad W_{c}(E)=A f(E),
$$

$W_{c}(E)$ имеет смысл вероятности обнаружить $\beta$-электрон в интервале энергий от $E$ до $E+d E$. Вероятность того, что электрон с энергией $E$, генерированный на глубине $z$, достигнет поверхности, равна

$$
W_{s}(E)=W_{c}(E) h\left(z / R_{c}(E)\right) .
$$

В формуле учтено, что импульс, направленный к поверхности, имеет половину всех электронов, однако,

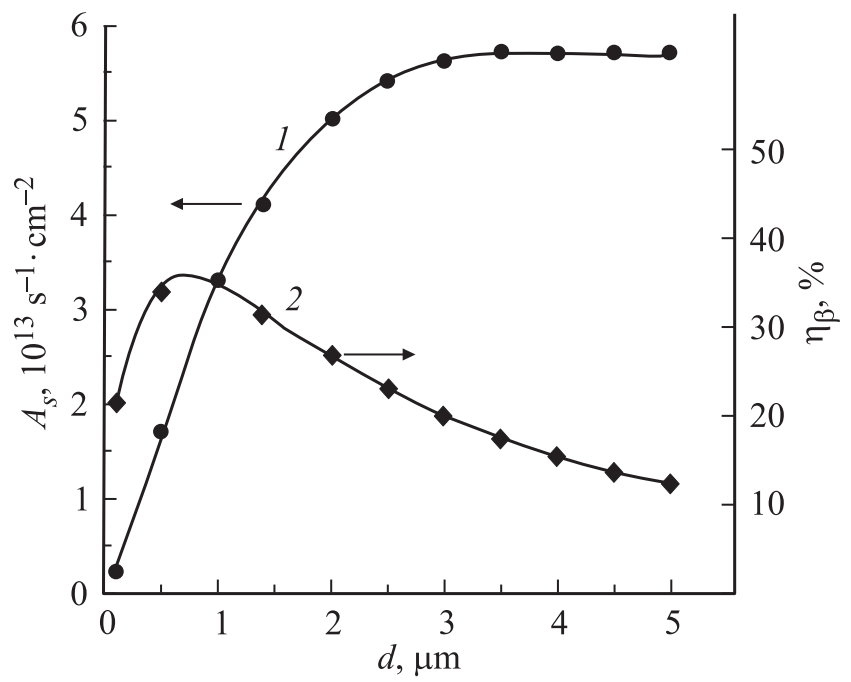

Рис. 2. Зависимость поверхностной активности (1) и коэффициента эффективности (2) источника излучения на основе Ni-63.

используются два полупроводниковых преобразователя. Структура имеет вид сандвича с излучателем между преобразователями. Вероятность того, что электрон любой энергии, генерированный на глубине $z$, достигнет поверхности, равна

$$
W_{s}=\int_{0}^{E_{\max }} W_{c}(E) h\left(z / R_{c}(E)\right) d E
$$

Тогда выражение для поверхностной активности принимает вид

$$
A_{s}=A_{0 V} S \int_{0}^{d} d z \int_{0}^{E_{\max }} W_{c}(E) h\left(z / R_{c}(E)\right) d E
$$

Полная активность $\beta$-источника $(A)$ растет пропорционально его толщине $d: A=A_{0 V} S d$. Отношение поверхностной активности (8) к полной равно коэффициенту эффективности источника $\eta_{\beta}$. Эта величина имеет максимум при некоторой толщине $d_{\text {opt }}$, которая является оптимальной толщиной $\beta$-источника. На рис. 2 приведены результаты расчета поверхностной активности и эффективности изотопа Ni-63 в зависимости от толщины слоя изотопа. Оптимальная толщина слоя изотопа, оцененная в данной работе, порядка 1 мкм. Вернемся к результатам, приведенным на рис. 1. Можно предположить, что насыщение величины тока может быть обусловлено тем, что толщина слоя изотопа с увеличением активности превышает оптималь-

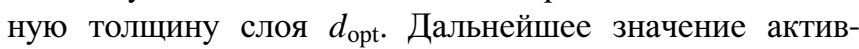
ности соответствуют объемной, а не поверхностной активности. 


\section{4. Время жизни неосновных носителей заряда в полупроводниковом преобразователе}

Выражения для плотности тока рекомбинации в области пространственного заряда (ОПЗ) $p-n$-перехода получены в работах $[14,15]$. В случае одного центра рекомбинации оно имеет вид

$$
j_{r}=\frac{2 k T w(U)}{\left(V_{d}-U\right)} \cdot \frac{c_{n} c_{p} n_{i}^{2} N_{t}\left[\exp \left(\frac{q U}{k T}\right)-1\right]}{2 n \sqrt{c_{n} c_{p}} \exp \left(\frac{q U}{2 k T}\right)+c_{n} n_{1}+c_{p} p_{1}},
$$

где $w$ - ширина ОПЗ; $V_{d}$ - диффузионный потенциал; $q$ - элементарный заряд; $c_{n}$ - коэффициент захвата электронов из зоны проводимости; $c_{p}$ - коэффициент захвата дырок; $U$ - напряжение смещения на области пространственного заряда диода; $k$ - постоянная Больцмана; $T$ - абсолютная температура; $N_{t}$ - концентрация центров рекомбинации; $n_{1}$ - концентрация электронов $\left(p_{1}\right.$ - дырок) в зоне проводимости (валентной зоне) при условии, что уровень Ферми совпадает с уровнем энергии рекомбинационного центра:

$$
n_{1}=N_{c} \exp \left(-\frac{E_{c}-E_{t}}{k T}\right) ; \quad p_{1}=N_{v} \exp \left(-\frac{E_{t}-E_{v}}{k T}\right) .
$$

Здесь $N_{c}$ - эффективная плотность состояний зоны проводимости, $N_{v}$ - эффективная плотность состояний валентной зоны, $E_{t}$ - энергия глубокого рекомбинационного центра, $E_{c}$ - энергия дна зоны проводимости, $E_{v}$ - энергия потолка валентной зоны.

Для вычисления параметров центров рекомбинации представим плотность рекомбинационного тока в области пространственного заряда в более простом виде. Преобразование вольт-амперной характеристики выполним путем деления (9) на $n_{i}, \frac{2 k T w(U)}{V_{d}-U}$ и $\exp \left(\frac{q U}{2 k T}\right)-1$. Выражение, полученное в результате деления, называется приведенной скоростью рекомбинации. Введем величины: $\tau_{n}^{-1}=c_{n} N_{t}, \tau_{p}^{-1}=c_{p} N_{t}$, где $\tau_{n}-$ время жизни при захвате электрона на пустой центр; $\tau_{p}$ время жизни при захвате дырки на рекомбинационный центр, полностью заполненный электронами. Примем для определенности, что глубокий уровень лежит выше запрещенной зоны, т.е. $c_{n} n_{1} \gg c_{p} p_{1}$. Тогда выражение для приведенной скорости рекомбинации имеет вид

$$
R_{\mathrm{prS}}=\frac{\frac{\tau_{n}^{-1} \tau_{p}^{-1}}{N_{t}} n_{i}\left[\exp \left(\frac{q U}{2 k T}\right)+1\right]}{\frac{2 n_{t} \sqrt{\tau_{n}^{-1} \tau_{p}^{-1}}}{N_{t}} \exp \left(\frac{q U}{2 k T}\right)+c_{n} n_{1}}
$$

График этой зависимости имеет вид ступеньки. Пренебрежем единицей в числителе. В случае, когда первое слагаемое в знаменателе много меньше второго:

$$
R_{\mathrm{prS}} \approx \tau_{p}^{-1} \frac{n_{i}}{n_{1}} \exp \left(\frac{q U}{2 k T}\right)
$$

Наоборот, когда первое слагаемое много больше второго, то

$$
R_{\mathrm{prS}} \approx \frac{\sqrt{\tau_{n}^{-1} \tau_{p}^{-1}}}{2}
$$

Выберем на зависимости приведенной скорости рекомбинации некоторую точку, с координатами $R_{1}, U_{1}$, тогда решая совместно систему уравнений (12)-(13), получаем для характеристических времен жизни следующие соотношения:

$$
\begin{gathered}
\tau_{n}^{-1}=\frac{4 R_{\mathrm{prS}}^{2} n_{i}}{n_{1} R_{1}} \exp \left(\frac{q U_{1}}{2 k T}\right), \\
\tau_{p}^{-1}=\frac{n_{1} R_{1}}{n_{i}} \exp \left(-\frac{q U_{1}}{2 k T}\right), \\
\frac{c_{p}}{c_{n}}=\frac{n_{1}^{2} R_{1}^{2}}{4 R_{\mathrm{prS}}^{2} n_{i}^{2}} \exp \left(-\frac{q U_{1}}{k T}\right) .
\end{gathered}
$$

В случае, когда первое и второе слагаемые в знаменателе (11) равны, например, при напряжении $U_{0}$, получается формула для вычисления энергии активации:

$$
E_{c}-E_{t}=\frac{E_{g}}{2}-\frac{q U_{0}}{2}+\frac{3}{4} k T \ln \left(\frac{m_{n}^{*}}{m_{p}^{*}}\right)+\frac{1}{2} k T \ln \left(4 \frac{\tau_{p}}{\tau_{n}}\right) .
$$

Здесь $E_{g}$ - ширина запрещенной зоны, $m_{n(p)}^{*}$ - эффективная масса электрона (дырки) в зоне проводимости (в валентной зоне).

В дальнейшем уравнения (14) и (15) использовались для анализа температурных зависимостей времени жизни. При каждой температуре было проведено разделение рекомбинационных процессов на составляющие и определены характеристические параметры рекомбинационных центров. Обработка экспериментальных вольт-

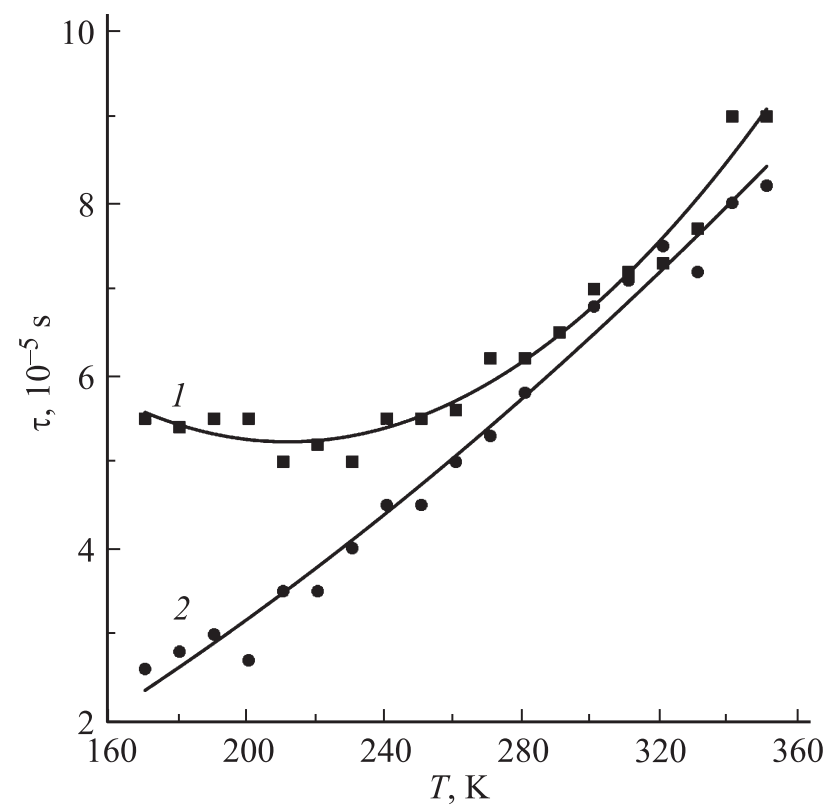

Рис. 3. Температурная зависимость времени жизни при рекомбинации с участием центров с энергией активации, эВ: $1-0.39,2-0.45$. 
амперных характеристик при различных температурах позволила выявить, что рекомбинация определяется Двумя центрами, энергии активации которых 0.39 и 0.45 эВ. Температурные зависимости времени жизни демонстрируют небольшой рост с повышением температуры (рис. 3). В целом рекомбинационные центры с энергиями 0.45 и 0.39 эВ имеют достаточно стабильные параметры, которые слабо зависят от температуры.

\section{5. Вычисление напряжения холостого хода, возникающего в полупроводниковом преобразователе при облучении его электронами высоких энергий}

При генерации электронов и дырок в $i$-области электронами высоких энергий возникает градиент концентрации носителей заряда, который вызывает диффузионные токи по направлению к поверхности. При диффузии электроны и дырки рекомбинируют посредством центров безызлучательной рекомбинации в объеме материала. Для получения теоретической величины напряжения холостого хода будем решать уравнение непрерывности при следующих допущениях.

1) Вследствие высокого уровня легирования $p$ - и $n$-областей, по сравнению с $i$-базой, через $p-i$-переход течет чисто дырочный ток, а через $n-i$-переход чисто электронный ток. Это допущение означает следующее. Поскольку избыточные неосновные носители заряда практически не проникают в $p$ - и $n$-области, то рекомбинацией электронно-дырочных пар в этих областях можно пренебречь.

2) В $i$-области реализуется высокий уровень инжекции носителей заряда, т.е. $n \gg n_{i}, p \gg n_{i}$. Падение напряжения на сопротивлениях $p$ - и $n$-областей из-за сильного легирования пренебрежимо мало. Полное падение напряжения на $p-i-n$-диоде складывается из падений напряжения на $p-i$-переходе, $n-i$-переходе и на $i$-области.

3) При высоком уровне инжекции время жизни носителей заряда в $i$-области $\tau=\tau_{\infty}$ и не зависит от концентрации носителей заряда $\left(\tau_{\infty}-\right.$ время жизни при высоком уровне возбуждения полупроводника). Это допущение справедливо при рекомбинации носителей заряда через рекомбинационные центры.

4) В $i$-области выполняется условие квазинейтральности $n(x)=p(x)$.

При таких допущениях уравнения для плотностей тока электронов и дырок и уравнения непрерывности $i$-области можно записать в виде:

$$
\begin{gathered}
J_{p}=q \mu_{p} p F-q D_{p} \frac{d p}{d x}, \quad J_{n}=q \mu_{n} p F+q D_{n} \frac{d p}{d x}, \\
-\frac{1}{q} \frac{d J_{p}}{d x}-\frac{p-n_{i}}{\tau}+G(x)=0, \frac{1}{q} \frac{d J_{n}}{d x}-\frac{p-n_{i}}{\tau}+G(x)=0 .
\end{gathered}
$$

Здесь $\mu_{n}-$ подвижность электронов, $\mu_{p}-$ подвижность дырок, $p$ - концентрация дырок, $F-$ напряженность

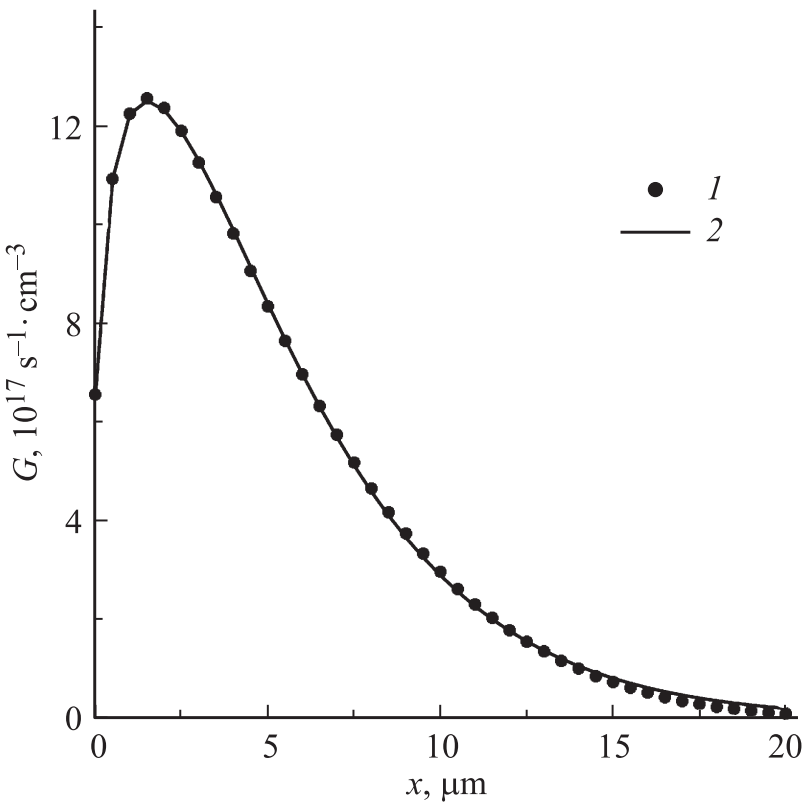

Рис. 4. Распределение электронов высоких энергий в области пространственного заряда $p-i-n$-диодов: 1 - расчет по формуле (18), 2 - аппроксимация расчета тремя экспоненциальными слагаемыми.

электрического поля в $i$-области, $J_{n(p)}-$ плотность электронного (дырочного) тока, $D_{n}-$ коэффициент диффузии электронов, $D_{p}-$ коэффициент диффузии дырок, $n_{i}$ - концентрация собственных носителей кремния, $G(x)$ - функция распределения генерированных электронов и дырок по $i$-области.

Электроны высоких энергий, вылетев из слоя изотопа, проникают в полупроводник и генерируют в нем вторичные электроны, которые обусловливают ток. Кроме того, необходимо учесть, что каждый $\beta$-электрон с энергией $E$ при взаимодействии с веществом полупроводника создает не одну, а сразу несколько электронно-дырочных пар. Поэтому в формулу для генерации необходимо ввести отношение энергии $\beta$-электрона к энергии образования электронно-дырочной пары в данном веществе. В связи со сказанным, для распределения функции генерации по ОПЗ необходимо использовать параметры полупроводникового слоя. Скорость генерации вторичных электронов на глубине $x$ определяется электронами всех энергий, которые вылетают из источника, поэтому

$$
G(x)=A_{s} \int_{0}^{E_{\max }} W_{c}(E) \frac{E}{E_{i}} h\left(\frac{x}{R_{\mathrm{ef}}(E)}\right) d E .
$$

Здесь $E_{i}=2.596 E_{g}+0.714=3.62$ эВ $=0.00362$ кэВ энергия образования электронно-дырочной пары в кремнии. В формулу (4), которая определяет $R_{\mathrm{ef}}(E)$, подставляется плотность кремния.

Выражение (18) определяет количество электронов, которые генерируются в одну секунду на глубине $x$. За- 
висимость $G(x)$ в общем случае может быть описана эмпирической функцией вида $G(x)=g_{1} \sum_{i=1}^{n} A_{i} \exp \left(-k_{i} x\right)$. Лучшее приближение к исходным точкам обеспечивает сумма трех экспонент. Поэтому в дальнейшем в качестве $G(x)$ будет использоваться сумма трех экспонент (рис. 4): $G(x)=g_{1}\left(A_{1} \exp \left(-k_{1} x\right)+A_{2} \exp \left(-k_{2} x\right)\right.$ $\left.+A_{3} \exp \left(-k_{3} x\right)\right)$, где $A_{i}, k_{i}$ - параметры экспонент $(i=1,2,3)$. Алгоритм расчета можно найти в работе [16]. Рассчитанная величина напряжения холостого хода имеет вид:

$$
\begin{aligned}
& U_{\mathrm{oc}}=\frac{k T}{q} \ln \left(\frac{1}{n_{i}^{2}}\left(n_{i}+\frac{L_{a}}{\operatorname{sh}\left(\frac{W}{L_{a}}\right)}\left[-\beta+\alpha \operatorname{ch}\left(\frac{W}{L_{a}}\right)\right]+\tilde{\alpha}\right)\right. \\
& \left.\times\left(n_{i}+\frac{L_{a}}{\operatorname{sh}\left(\frac{W}{L_{a}}\right)}\left[-\beta \operatorname{ch}\left(\frac{W}{L_{a}}\right)+\alpha\right]+\tilde{\beta}\right)\right) \\
& +\frac{D_{n}-D_{p}}{\mu_{p}+\mu_{n}} \ln \left(\frac{n_{i}+\frac{L_{a}}{\operatorname{sh}\left(\frac{W}{L_{a}}\right)}\left[-\beta+\alpha \operatorname{ch}\left(\frac{W}{L_{a}}\right)\right]+\tilde{a}}{n_{i}+\frac{L_{a}}{\operatorname{sh}\left(\frac{W}{L_{a}}\right)}\left[-\beta \operatorname{ch}\left(\frac{W}{L_{a}}\right)+\alpha\right]+\tilde{\beta}}\right),
\end{aligned}
$$

где $L_{a}=\left(D_{a} \tau\right)^{1 / 2}-$ амбиполярная диффузионная длина, $D_{a}=\frac{2 k T \mu_{p} \mu_{n}}{q\left(\mu_{p}+\mu_{n}\right)}-$ амбиполярный коэффициент диффузии, $W$ - ширина $i$-области, остальные коэффициенты:

$$
\begin{aligned}
& \left\{\begin{array}{l}
\alpha=\frac{g_{1} L_{a}^{2}}{D_{a}} \sum_{i=1}^{n} \frac{-k_{i} A_{i}}{1-L_{a}^{2} k_{i}^{2}}, \\
\beta=\frac{g_{1} L_{a}^{2}}{D_{a}} \sum_{i=1}^{n} \frac{-k_{i} A_{i} \exp \left(-k_{i} W\right)}{1-L_{a}^{2} k_{i}^{2}},
\end{array}\right. \\
& \left\{\begin{array}{l}
\tilde{\alpha}=\frac{g_{1} L_{a}^{2}}{D_{a}} \sum_{i=1}^{n} \frac{A_{i}}{1-L_{a}^{2} k_{i}^{2}}, \\
\tilde{\beta}=\frac{g_{1} L_{a}^{2}}{D_{a}} \sum_{i=1}^{n} \frac{A_{i} \exp \left(-k_{i} W\right)}{1-L_{a}^{2} k_{i}^{2}} .
\end{array}\right.
\end{aligned}
$$

Для расчета напряжения холостого хода использовались параметры рекомбинационных центров, вычисленные в предыдущем пункте. Максимальное значение напряжения холостого хода, которое соответствует выходу электронов из слоя оптимальной толщины, для изотопа Ni-63 равно $U_{\text {oc }}=0.26 \mathrm{~B}$. Реальные поверхностные активности меньше. Расчет для никеля с различными значениями активности приведен на рис. 1. Экспериментальные значения на всем промежутке изменения активности составляют 50\% от теоретической величины. Это различие дает возможность оценить $\eta_{c}-$ долю электронов и дырок, которые попадают в область пространственного заряда и способны внести вклад в электрический ток. По-видимому, половина $\beta$-электронов отражается от поверхности полупроводника и они не могут участвовать в генерации тока.

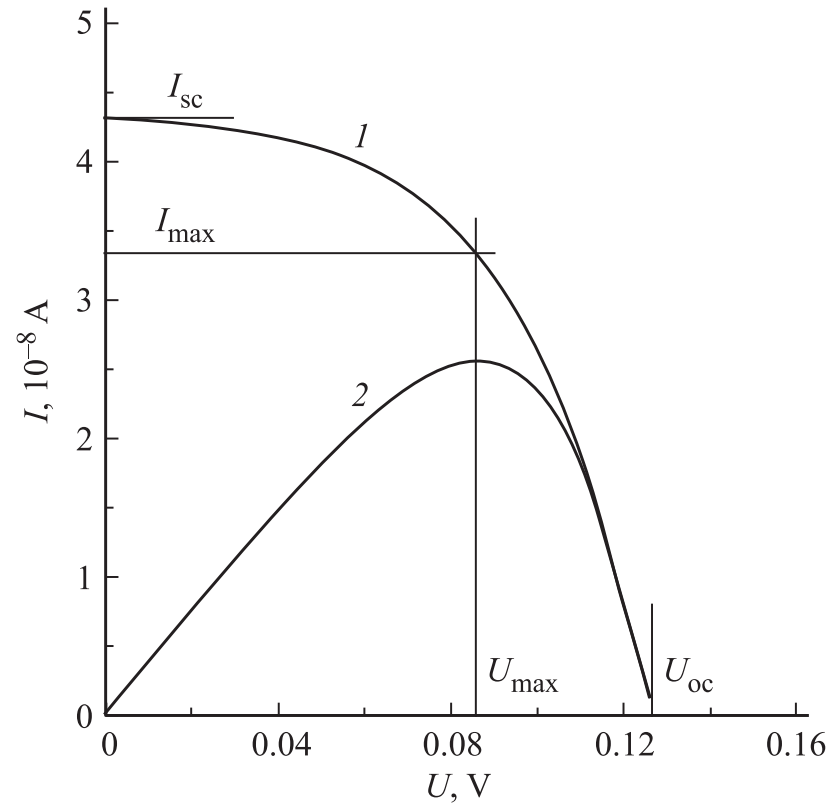

Рис. 5. Вольт-амперная характеристика (1) и кривая мощности на сопротивлении нагрузки (2) $p-i-n$-диода, облучаемого источником $\beta$-излучения.

\section{6. Интегральная эффективность полупроводникового преобразователя}

Интегральная эффективность полупроводникового преобразователя может быть вычислена по формуле $[10,16]$

$$
\eta_{s}=q U_{\mathrm{oc}} F_{\mathrm{ef}} / E_{i},
$$

где $E_{\mathrm{ef}}-$ фактор эффективности преобразования, $E_{i}-$ энергия, необходимая для создания одной электроннодырочной пары в преобразователе. Фактор эффективности $F_{\mathrm{ef}}$ можно определить по формуле

$$
F_{\mathrm{ef}}=\frac{I_{\mathrm{max}} U_{\mathrm{max}}}{I_{\mathrm{sc}} U_{\mathrm{oc}}},
$$

где $U_{\max }$ - напряжение, соответствующее максимуму мощности на нагрузочном сопротивлении; $I_{\max }$ - ток, соответствующий данному напряжению. Для того чтобы определить эти величины, на график тока генерации $\beta$-электронами нанесем мощность в относительных единицах (рис. 5). Определим напряжение, при котором мощность на сопротивлении нагрузки достигает максимума. От значения данного напряжения на оси напряжений проведем вертикальную линию до пересечения с вольт-амперной характеристикой. Тем самым найдем значение тока, соответствующее напряжению, при котором мощность максимальна. Далее вычисляем фактор эффективности по формуле (2). Для преобразователей, которые используются в данной работе, он равен 0.6. Эту величину будем использовать при дальнейших расчетах. 
Интегральная эффективность полупроводникового преобразователя, вычисленная по формуле (20), равна $\eta_{s}=2.5 \%$. Эта величина растет с увеличением напряжения холостого хода и уменьшается с ростом ширины запрещенной зоны полупроводника.

Произведение коэффициентов эффективности источника, собирания электронов и интегральной эффективности полупроводникового преобразователя дают теоретическое значение коэффициента полезного действия прямого преобразователя тока на основе изотопа Ni-63. В нашем случае эта величина составляет $0.8 \%$ и соответствует экспериментальным значениям, достигнутым в работе: $0.4-0.7 \%$.

Для дальнейшено повышения эффективности преобразователей необходимо повышать напряжение холостого хода. Эту проблему можно решить путем перехода к полупроводниковым преобразователям из более широкозонных материалов, либо созданием гетеропереходов кремния с карбидом кремния.

\section{7. Заключение}

В данной работе разработана технология создания высокоэффективных полупроводниковых преобразователей энергии $\beta$-распада в электрическую энергию, а также расчетная математическая модель самопоглощения электронов высоких энергий в веществе с учетом экспериментальных распределений этих электронов по энергиям. Оценен коэффициент полезного использования изотопов для радиоизотопных источников и преобразователей радиационной энергии в электрическую с учетом реальных энергий электронов, возникающих при распаде. Разработана методика определения оптимальных толщин активных слоев источников и преобразователей. В результате получен теоретический кпд $\beta$-батареек на основе пары: источник излучения $\mathrm{Ni}$-63 и полупроводниковый преобразователь - кремниевый $p-i-n$-диод. Проверка разработанных моделей по экспериментальным данным позволяет сделать вывод об адекватности предложенных моделей и возможности их использования для широкого круга $\beta$-источников.

Работа выполнена при поддержке Министерства образования и науки РФ в рамках государственной поддержки научных исследований.

\section{Список литературы}

[1] Polymers, phosphors, and voltaics for radioisotope microbatteries (London-N.Y.-Washington, CRC Press LLC, 2002).

[2] А.А. Резнев, А.А. Пустовалов, Е.М. Максимов, Н.К. Передерий, Н.С. Петренко. Нано- и микросистемная техника, 3 (104), 14 (2009).

[3] M.V.S. Chandrashekhar, Ch.I. Thomas, H. Li, M.G. Spencer, A. Lal. Appl. Phys. Lett., 88, 033506 (2006).
[4] C.J. Eiting, V. Krishnamoorthy, E. Romero, S. Jones. Proc. 42 Power Source Conf., 601 (2006).

[5] V.M. Andreev, A.G. Kavetsky, V.S. Khvostikov, V.R. Larionov, V.D. Rumyantsev, M.Z. Shvarts, E.V. Yakimova, V.A. Ystinov. Conf. Record of the Twenty-Eighth IEEE Photovoltaic Specialists Conference-2000, 1253 (2000).

[6] G.C. Rybicki. NASA Rev., 11, 200 (2001).

[7] H. Guo, A. Lal. IEEE Transducer, 36, 131 (2003).

[8] W. Sun, N.P. Kherani, K.D. Hirschman, L.L. Gadeken, P.M. Fauchet. Adv. Mater., 17 (10), 1230 (2005).

[9] J.K. Chu, X.G. Piao, M.J. Li et al. J. Micro/Nanolithograph MEMS-MOEMS, 8 (2), 021180 (2009).

[10] С.М. Зи. Физика полупроводниковых приборов (М., Мир, 1984) т. 1.

[11] T.E. Everhart, P.H. Hoff. J. Appl. Phys. 42, 5837 (1971).

[12] V.K.S. Ong, P.C. Phua. Semicond. Sci. Technol., 16, 691 (2001).

[13] В.М. Колобашкин, П.М. Рубцов, В.Г. Алексакин, А. Ружанский. Бета-излучение продуктов деления: Справочник (М., Атомиздат, 1978).

[14] С.В. Булярский, Н.С. Грушко. Генерационно-рекомбинационные процессы в активных элементах (М., МГУ, 1995).

[15] С.В. Булярский, Н.С. Грушко, А.И. Сомов, А.В. Лакалин. ФТП, 31 (9), 1146 (1997).

[16] В.И. Гаман. Физика полупроводниковых приборов (Томск, изд-во НТЛ, 2000).

Редактор А.Н. Смирнов

\section{Optimization of parameters of power sources excited by the $\beta$-radiation}

\author{
S.V. Bulyarsky ${ }^{1}$, A.V. Lakalin ${ }^{1}$, I.E. Abanin ${ }^{2}$, \\ V.V. Amelichev ${ }^{2}$, V.V. Svetuhin ${ }^{3}$ \\ ${ }^{1}$ Institute of Nanotechnology Microelectronics \\ Russian Academy of Sciences, \\ 119991 Moscow, Russia \\ ${ }^{2}$ NPK Technological Centre, \\ 124498 Moscow, Russia \\ ${ }^{3}$ Ulyanovsk State University, \\ 432000 Ulyanovsk, Russia
}

\begin{abstract}
The results of experimental studies and calculations of the energy conversion efficiency of the radiation $\beta$-source Ni-63 into electricity using silicon $p-i-n$-diodes. All calculations are made taking into account the distribution of $\beta$-electron energy. An expression for the open-circuit voltage converter based on the distribution of high-energy electrons in the space-charge $p-i-n$-diode. The ways of optimizing the parameters of the converter by improving the technology of diodes, to optimize the thickness of the active layer of the emitter and space charge region of the diodes. Calculated distribution of losses on the transformation of the source and receiver of radiation, as well as losses at the entrance of high-energy electrons in the semiconductor. The experimental values of the conversion efficiency is $0.4-0.7 \%$. These values are in good agreement with the calculated parameters.
\end{abstract}

